\title{
Tendinopatía de De Quervain (1). Revisión de conceptos
}

\section{G. Celester Barreiro}

Servicio de Cirugía de la Mano del Hospital Modelo. Mutua La Fraternidad. A Coruña ESPECIALISTA EN CIRUGÍA ORTOPÉDICA Y TRAUMATOLOGÍa

Correspondencia:

Dr. Gabriel Celester Barreiro

Avda. del Ejército 12, bajo (Mutua)

15006 A Coruña. Telf. 981296588

e-mail: gcelester@fraternidad.com

Objetivo: Existen algunos tópicos comúnmente aceptados sobre los conceptos básicos de la tendinopatía de de Quervain que conviene revisar de cuando en cuando, especialmente con respecto a la etiopatogenia, para así poder evaluar el estado actual de nuestros conocimientos sobre esta patología.

Palabras clave: Enfermedad de De Quervain, Tendinopatía estenosante de De Quervain, Tenosinovitis de De Quervain, Síndrome de Intersección.
Purpose: There exist some topics commonly accepted on the basic concepts of the De Quervain's disease that it suits to check every now and then, specially with regard to the etiology and pathogeny, in order to evaluate the present situation of our knowledge on this pathology.

Key words: De Quervain's disease, De Quervain's stenosing tendovaginitis, De Quervain's Tenosynovitis, Intersection Syndrome.

\section{INTRODUCCIÓN}

$\mathrm{E}$ n 1895, el cirujano Fritz de Quervain ${ }^{1}$ (de verdadero nombre Friedrich, que él mismo cambió por el de Fritz, con el que sería conocido en la literatura médica ${ }^{2}$ ), publicó cinco casos de afectación del extensor pollicis brevis (EPB) y del abductor pollicis longus (APL) en el primer compartimento extensor de la muñeca, llamándole tendovaginitis crónica seca para diferenciarla de las formas inflamatorias y sépticas.

Algunos autores de lengua inglesa ${ }^{3-5}$ afirman que el mismo cuado clínico había sido previamente descrito dos años antes en la $13^{\mathrm{a}}$ edición de la Anatomy de Henry Gray. Desde luego no pudo haber sido descrito por el propio Gray, que había fallecido 34 años antes de esta edición, en 1861. Pero, en cualquier caso, desde la publica- ción del citado artículo europeo, esta entidad patológica se conoce con el epónimo del cirujano suizo, discípulo de Kocher, como Tendinopatía de De Quervain.

La afectación de las vainas puede deberse a una inflamación aguda (tendinitis) o, más frecuentemente, a su irritación crónica (tendinosis), por lo que, en rigor, el cuadro que engloba ambas formas debería ser denominado tendinopatía del primer compartimento extensor de la muñeca, o, si se prefiere, Tendinopatía de De Quervain (TDQ).

\section{OBJETIVO}

Existen algunos tópicos comúnmente aceptados sobre los conceptos básicos de la TDQ que conviene revisar de cuando en cuando, especialmente con respecto a la etiopatogenia. 
El objetivo de este artículo de revisión de conceptos es evaluar el estado actual de nuestros conocimientos sobre esta patología.

\section{RECUERDO ANATOMOFISIOLÓGICO}

El lecho del primer compartimento extensor de la muñeca, situado sobre el borde de la apófisis estiloides del radio, es liso, ligeramente acanalado en sentido transversal, y oblicuo de dentro afuera y de proximal a distal con respecto al eje longitudinal del antebrazo. Suele estar dividido a lo largo por un tabique fibroso que separa el extensor pollicis brevis (EPB) de los tendones accesorios - casi siempre varios - del abductor pollicis longus (APL).

Este canal está techado por un retináculo conjuntival cuya función es actuar de fulcro, permitiendo el cambio de dirección de los tendones al tiempo que impide su desplazamiento lateral ${ }^{4}$. Para favorecer el deslizamiento, los tendones están recubiertos por una membrana sinovial que se prolonga alrededor de $1 \mathrm{~cm}$ fuera del canal en ambos sentidos.

Tanto los tendones extensores de los dedos trifalángicos como los extensores radiales de la muñeca (extensor digitorum, extensor carpi radialis brevis y extensor carpi radialis longus) nacen en la zona epicondílea del codo y siguen, por tanto, la dirección longitudinal del antebrazo. Pero los extensores del pulgar tienen una trayectoria muy oblicua dado que el primer metacarpiano forma con el antebrazo un ángulo de unos $40^{\circ}-50^{\circ}$ cuando el pulgar está en abducción y extensión completas. De ahí la importancia de esta primera polea como fulcro.

\section{ETIOPATOGENIA}

Habitualmente, la TDQ es de origen mecánico por la irritación de las vainas tendinosas debido a su fricción dentro del canal osteofibroso. Suele ser por la ejecución de pinzas de fuerza entre el pulgar y el índice - como ocurre en las etiquetadoras de la industria textil-, por la flexo-extensión reiterada de la articulación trapeciometacarpiana - propio de las plancha-

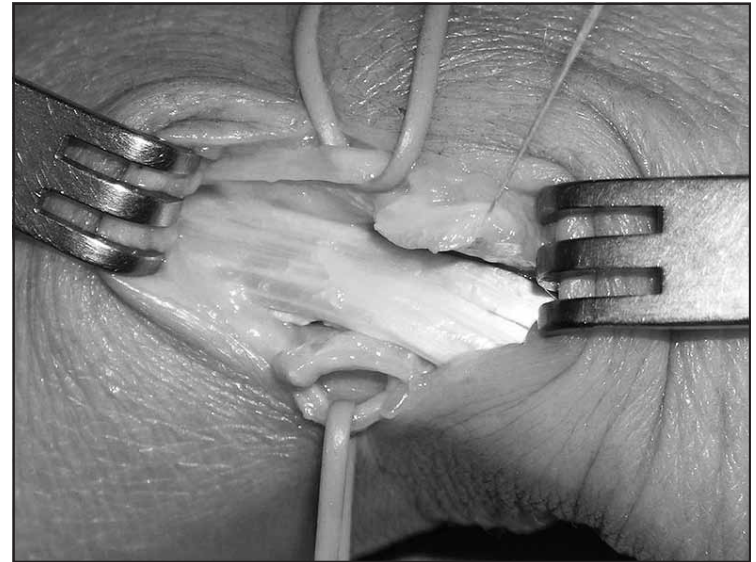

Figura 1: Polea muy engrosada. Apréciese que tiene el mismo grosor, o más, que las ramas del nervio radial.

doras industriales, en cuyas planchas el botón de vapor sólo puede ser accionado con el pulgar de la mano derecha-, o de la articulación radiocarpiana por maniobras repetitivas de la muñeca en desviación radial-cubital, como ocurre en las carniceras, pintores o chapistas.

Dicho de otro modo, existen dos mecanismos de producción, uno estático, por el empleo continuo de pinzas de fuerza entre el índice y el pulgar, aun con poca o ninguna movilidad, como es la maniobra de introducir una fuente en el microondas o bandejas en los hornos eléctricos de las panaderías; y otro dinámico, por la reiterada movilidad de la articulación trapeciometacarpiana, con el uso de las tijeras de podar por ejemplo, o de la radiocarpiana, como en la acción de martillear.

Probablemente, en las personas que realizan movimientos repetitivos de abducción del pulgar los tendones ejercen la presión sobre el techo del retináculo produciendo un engrosamiento del mismo, lo que conllevará a un cuadro estenosante por disminución de la luz del canal. De ahí que en algunos pacientes con mucho tiempo de evolución suelan encontrarse poleas muy endurecidas y con varios milímetros de grosor (Figura 1). Por el contrario, en otros pacientes cuya fuerza predomina sobre el lecho óseo, la fricción causa una fibrosis defensiva de las vainas sinoviales, lo que aumenta el volumen dentro del túnel. De hecho, en algunos pacientes se aprecia una polea normal pero con una notoria hipertrofia de las vainas sinoviales del EPB y APL (Figuras 2A y 2B). 

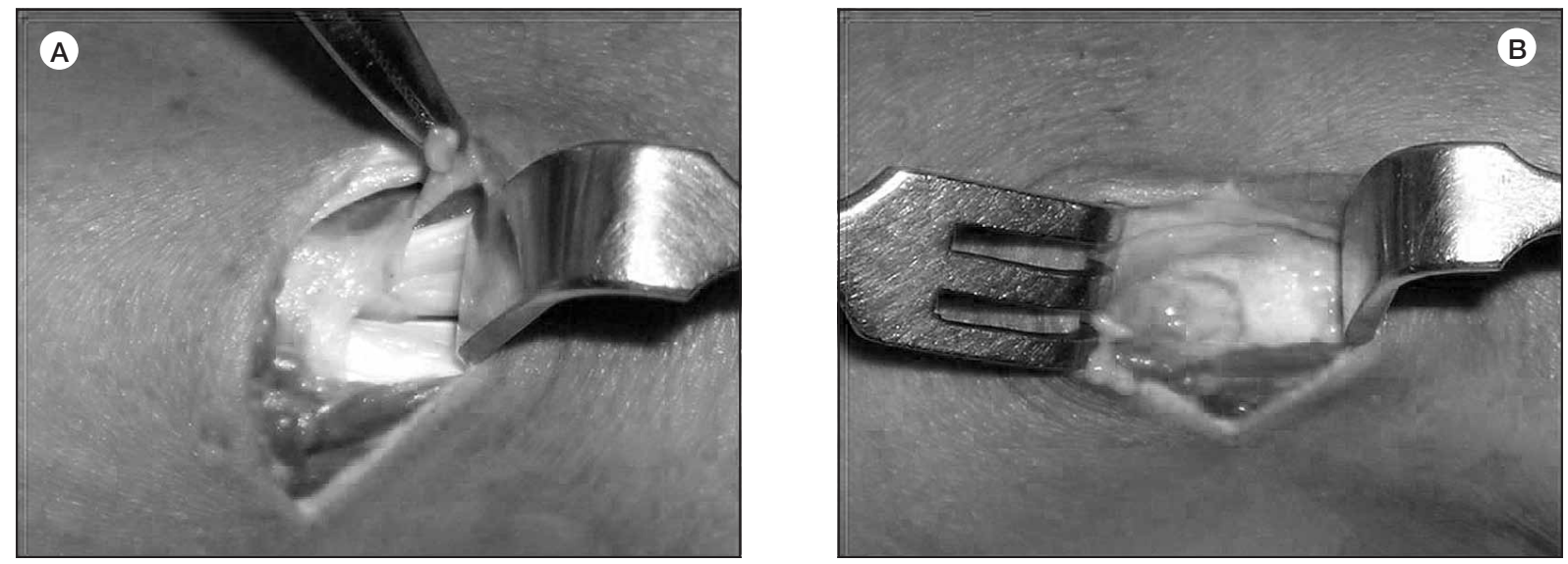

Figura 2, A y B: Sinovial hipertrófica del primer compartimento extensor de la muñeca. A: en la parte proximal. B: en la distal.

Por supuesto, existen numerosas ocupaciones en las que se dan conjuntamente ambos mecanismos, pero en cualquier caso la anatomía patológica está en la polea, que se hipertrofia, o en la vaina sinovial por edema y fibrosis, no en los propios tendones, cuyo aspecto es siempre normal.

Por esta frecuente relación con el trabajo, la TDQ está contemplada en la legislación española como una enfermedad profesional en aquellas ocupaciones de fuerza o de movimientos repetitivos de la mano bajo el epígrafe de Enfermedades por fatiga de las vainas tendinosas, tejidos peritendinosos e inserciones musculares.

Menos frecuente es encontrar una TDQ de origen inflamatorio por enfermedades sistémicas del tejido conjuntivo, como la artritis reumatoide, ya que estos procesos disminuyen la movilidad trapeciometacarpiana y radiocarpiana, cuyo excesivo uso parece el principal factor predisponente. Y aún más rara vez puede ser debida a un ganglión de la vaina sinovial. Phalen ${ }^{3}$ señala que a veces puede palparse un pequeño ganglión adyacente al borde del primer compartimento dorsal, pero no es fácil establecer si el ganglión causa la enfermedad o es una consecuencia de la misma debido a la degeneración de las vainas tendinosas, como se aprecia en la Figura 3.

La TDQ puede tener un origen iatrogénico tras el tratamiento quirúrgico de la rizartrosis con tenoplastias que realizan una lazada del APL alrededor o a través del flexor carpi radialis, aumentando la presión del APL contra su lecho óseo y por tanto la fricción. Aunque este último mecanismo ha sido descrito, Ceruso et al. ${ }^{6}$ consideran superfluo abrir el primer compartimento al operar de este modo las rizartrosis porque al emplear un tendón accesorio del APL para la tenoplastia ya disminuye el volumen tendinoso intracanalar. También puede provocarse una TDQ iatrogénica al realizar la osteosíntesis de las fracturas de la extremidad distal del radio con placas con una pestaña que asienta en el lecho del primer compartimento, como se aprecia en la Figura 4, radiografía de un paciente al que debió retirársele la placa por este motivo.

Sin duda, en la etiopatogenia de la TDQ también juegan un papel importante las frecuentes variantes anatómicas de esta zona anatómica, tanto en su aspecto osteofibroso, cuando está muy engrosado el tabique longitudinal que di-

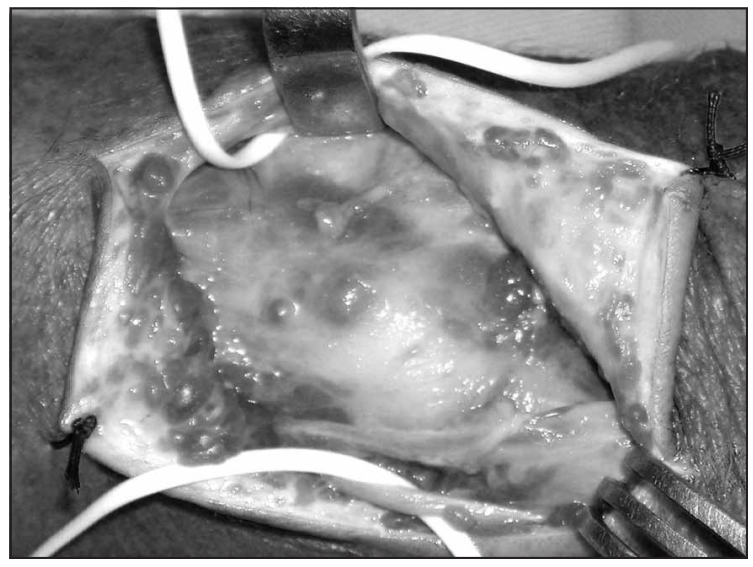

Figura 3: Ganglión bilobulado en la entrada del primer compartimento extensor. 


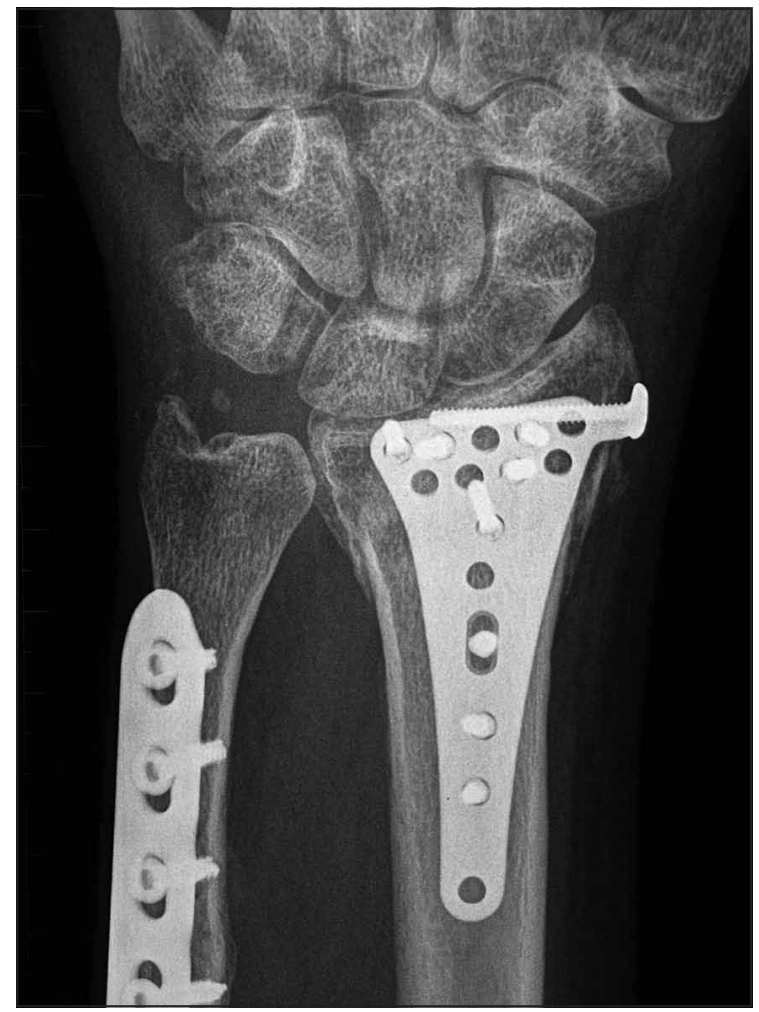

Figura 4: Radiografía de un paciente con $T D Q$ por ocupación del primer compartimento extensor con una placa metálica.

vide el compartimento en dos túneles ${ }^{3}$ (Figura 5A), como tendinoso, por el aumento de volumen dentro del canal debido a un gran número de tendones accesorios del APL (Zancolli y Cozzi $^{7}$ los hallan en el $100 \%$ de sus pacientes operados de rizartrosis), por las anomalías de inserción de este tendón (para Zancolli más del $70 \%$ se insertan en el opponens pollicis mientras Bouchlis et al. ${ }^{8}$ encuentran que en el $42 \%$ los fascículos más anteriores del APL se insertan en el abductor pollicis brevis) o por un implante muy distal de los vientres musculares, que llegan a entrar en el compartimento (Figura 5B).

Además de esta gran variedad de inserción de los tendones del APL, la dirección oblicua del túnel como factor coadyuvante de la TDQ parece demostrarlo el hecho de que esta tendinopatía sea mucho más frecuente que las de los demás tendones extensores de la muñeca y dedos. Pero, en rigor, se desconoce el papel que pueda jugar la dirección que tomen esos tendones al abandonar el primer compartimento para implantarse en la base del metacarpiano, en el trapecio o en los músculos de la región tenar. De hecho, la TDQ no es frecuente tras las consolidaciones defectuosas de la extremidad distal del radio pese a que éstas alteran a veces notoriamente la dirección tendinosa.

\section{EPIDEMIOLOGÍA}

Todos los autores coinciden en que la incidencia es mayor en mujeres ${ }^{3-5}$. Algunos señalan una frecuencia más alta en la quinta y sexta décadas de la vida ${ }^{5}$ y es probable que así haya sido en la población general hasta hace unos años, pero desde la incorporación masiva de la mujer al mercado laboral, especialmente a determinados

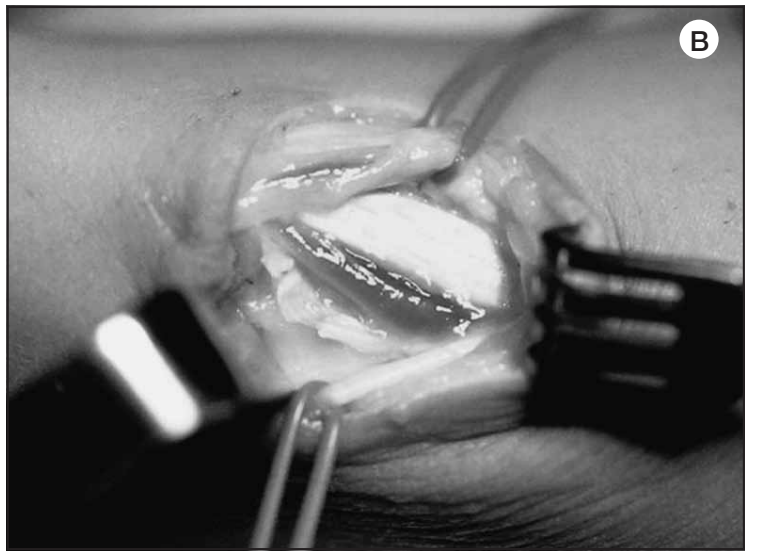

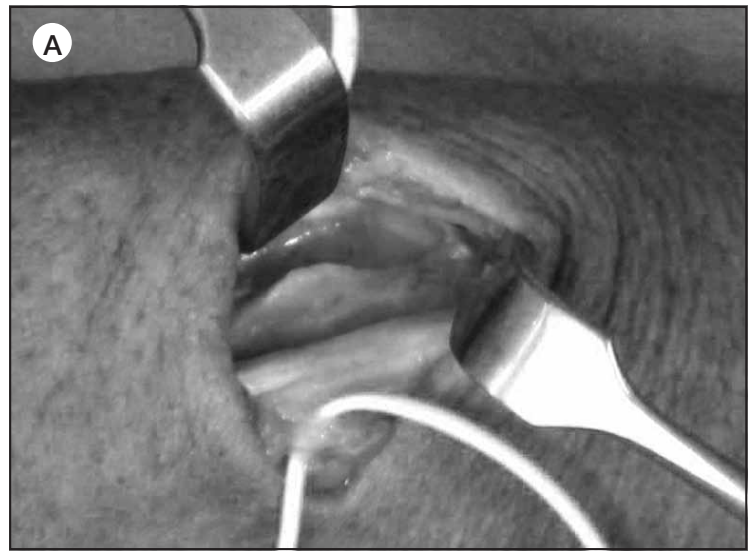

Figura 5: Factores anatómicos favorecedores de la tendinopatía. 5-A: Grueso tabique que divide longitudinalmente el compartimento extensor en dos canales, uno para el EPB y ótro para los tendones accesorios del APL. 5-B: Vientre muscular del EPB dientro del canal. 


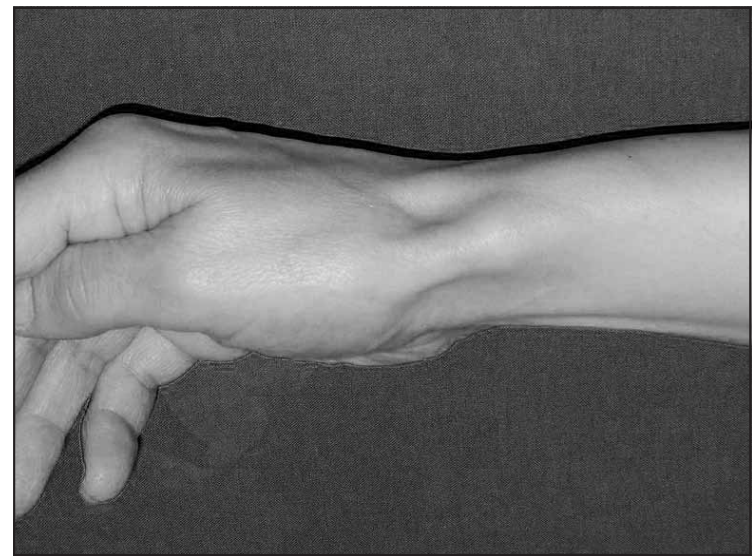

Figura 6: Notoria prominencia sobre la apófisis estiloides radial por el aumento de volumen de la polea del primer compartimento extensor.

trabajos, puede apreciarse en los últimos tiempos una incidencia mayor en mujeres jóvenes con profesiones que exigen reiteradas pinzas de fuerza, como carniceras o charcuteras ${ }^{9-11}$, al margen de su mayor incidencia en mujeres embarazadas $\mathrm{y}$ en los pacientes tratados con fluorquinolonas.

\section{DIAGNÓSTICO}

El diagnóstico de la TDQ se hace por la anamnesis y la exploración clínica. El paciente suele referir dolor mal localizado en el borde radial de la muñeca o de la mano. Con frecuencia puede encontrarse hinchazón local sobre la apófisis estiloides por el engrosamiento de la polea (Figura 6) o una deformidad fusiforme sobre los tendones cuando es notoria la proliferación tenosinovial. La presión sobre esa zona despierta dolor.

La maniobra de Finkelstein ${ }^{12}$, de inclinación cubital de la muñeca con el pulgar en flexión completa, despierta un dolor muy vivo. Brune$11 i^{13}$ describió la maniobra contraria, de extensión completa del pulgar con la muñeca en inclinación radial, y afirma que este signo es mucho más específico (Figura 7). En realidad, una maniobra similar a la de Finkelstein había sido ya descrita por Eichhoff ${ }^{14}$ en 1927, forzando la flexión del pulgar entre los demás dedos (Figura 8). Elliott ${ }^{15}$ destacó la amplia confusión de ambas maniobras en la literatura médica y que la de Eichhoff con frecuencia produce falsos positivos ya que el exceso de flexión de la tra-

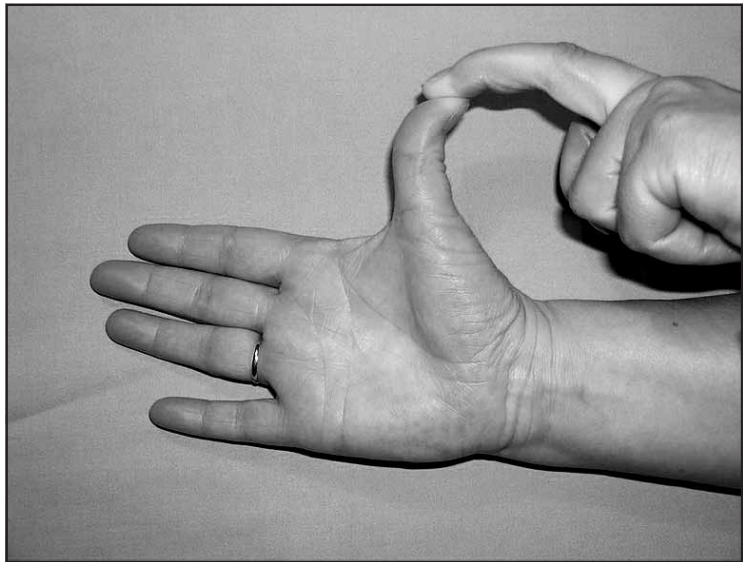

Figura 7: Maniobra de Brunelli.

peciometacarpiana puede provocar dolor incluso en personas sanas. Por otra parte, una maniobra muy similar a la de Brunelli ${ }^{13}$ (la provocación de dolor por extensión y abducción del pulgar) la describió Phalen ${ }^{3}$ en el tratado de Flynn publicado en 1984. El mecanismo de provocación de dolor entre ambas maniobras es claramente distinto; en la de Finkelstein los tendones ejercen la presión sobre el lecho óseo y en la de Brunelli sobre el techo fibroso.

Aunque la maniobra de Finkelstein también puede producir falsos positivos y depende del grado de extensión de la muñeca porque es más dependiente de la tracción del EPB que del $\mathrm{APL}^{16}$, coincidimos con Le $\operatorname{Viet}^{17}$ en que esta prueba es casi patognomónica de TDQ y diferimos con Brunelli. En nuestra experiencia personal el signo de Finkelstein resultó mucho más fidedigno, siendo positivo en el $98 \%$ de los pacientes ( $92 \%$ en la serie de Arroyo et al. ${ }^{9}$ ), mientras que el de Brunelli sólo lo fue en el $43 \%$.

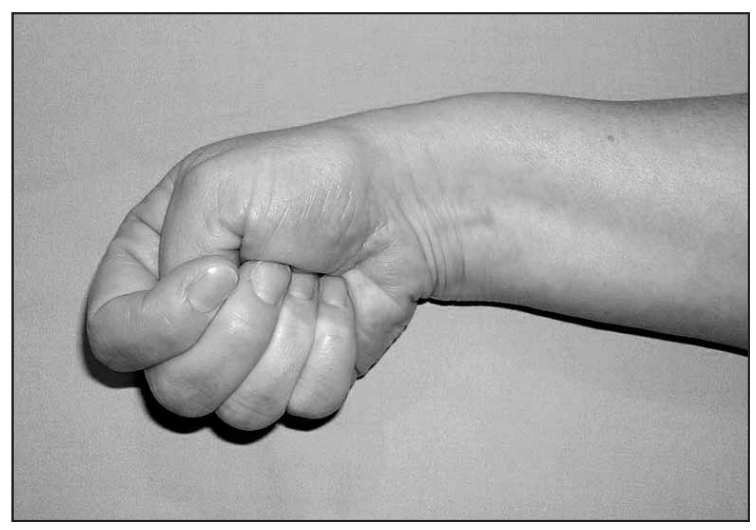

Figura 8: Maniobra de Eichhoff. 


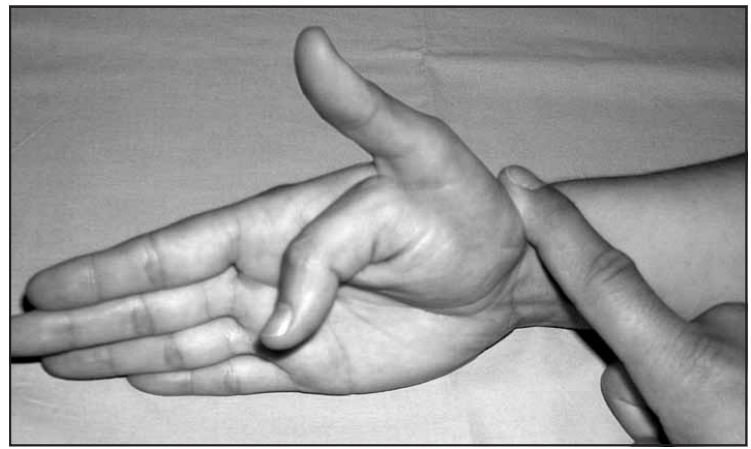

Figura 9: Maniobra del autor. Con el dedo del explorador sobre los tendones del primer compartimento, a su paso sobre el escafoides y trapecio, se le pide al paciente que extienda y flexione completa y repetidamente el dedo pulgar.

Desde hace más de un año venimos realizando una maniobra dinámica que no hemos visto descrita en la bibliografía consultada: con el antebrazo del paciente apoyado en la mesa por su borde cubital, ponemos un dedo sobre los tendones del primer compartimento en el hueco que hay entre el extremo de la apófisis estiloides y la base del primer metacarpiano. Ejerciendo presión con el dedo se le pide al paciente que realice repetidos movimientos de flexoextensión lo más amplios posible. La maniobra se considera positiva si despierta dolor y negativa si es indolora. En ningún caso esta maniobra resultó positiva en muñecas sanas, y sí en todos los pacientes con TDQ (Figura 9). Por supuesto, podría realizarse la maniobra con la muñeca del paciente suspendida en el aire, fijada entre los dedos índice y pulgar del explorador. Pero el hecho de apoyarla en la mesa impide la desviación cubital, pues, como bien señala Brunelli ${ }^{13}$, esta desviación forzada tensa el ligamento colateral radial y los ligamentos trapecioescafoideos y trapeciometacarpianos, lo que puede provocar dolor aun en ausencia de una TDQ y por tanto un falso positivo en las maniobras de Eichhoff y Finkelstein.

En las TDQ aisladas, por otra parte, no suele encontrarse crepitación, y sí en algunos casos de concomitancia con Síndrome de Intersección (SI); pero en este caso el crujido no se percibe sobre la estiloides radial sino en la zona en que el APL y EPB cruzan sobre los extensores radiales de la muñeca, a unos 3 ó $4 \mathrm{~cm}$ de la interlínea radiocarpiana, donde a veces se puede notar la zona tumefacta.
Pruebas exploratorias: Aunque algunos autores aconsejan la petición sistemática de radiografías simples de muñeca ${ }^{5,18}$, éstas no suelen mostrar ninguna anormalidad. No obstante, deben pedirse cuando se aprecia una prominencia sobre la estiloides radial para descartar la existencia de una exostosis u otra alteración no detectable por la exploración clínica.

En el borde externo de la extremidad distal del radio se encuentran a menudo hallazgos de difícil interpretación. Tras estudiar 100 radiografías consecutivas de muñeca en pacientes sin diagnostico de TDQ encontramos que 72 presentaban el borde liso, 20 tenían un surco más o menos pronunciado y en 8 era dudoso manifestar si había surco o no. Además se constató que en los pacientes menores de 30 años el borde externo de la apófisis estiloides del radio suele ser liso y uniforme, mientras que en los pacientes añosos, especialmente en los que tuvieron trabajos de fuerza, este reborde es irregular bien en forma de entrante, como una muesca, o de saliente a modo de cresta o protuberancia, y depende mucho de la posición radiológica.

Sólo cuando sospechásemos una tumoración de partes blandas ${ }^{3} \mathrm{o}$ una alteración ósea estaría justificado pedir una ecografía, TAC o RM.

La gammagrafía con tecnecio suele ser negativa $^{5}$, y la ecografía sólo sirve para confirmar el diagnóstico clínico.

\section{DIAGNÓSTICO DIFERENCIAL}

El dolor en el lado radial de la muñeca puede tener múltiples causas, siendo las siguientes las más frecuentes:

a) Origen óseo: estiloiditis radial por exostosis o reacciones periósticas, consolidaciones en posición viciosa de la extremidad distal del radio y seudoartrosis de escafoides ${ }^{3,4}$.

b) Origen articular: con malformaciones congénitas ${ }^{19}$, esguinces de los ligamentos trapeciometacarpianos y artrosis en el lado radial del carpo (escafoestiloidea, trapecioescafoidea o trapeciometacarpiana), que pueden coexistir por tener características demográficas similares $^{5}$. Los estudios radiológicos y una exploración clínica rigurosa suelen establecer el diagnóstico correcto. 
c) Origen tendinoso:

- Tendinitis del brachioradialis: el dolor suele acentuarse con la supinación contrariada.

- Tendinitis del extensor pollicis longus: si este tendón discurre por fuera de su corredera habitual, su tenosinovitis puede prestarse a confusión ${ }^{20}$.

- Síndrome de Intersección: el dolor y la crepitación al presionar sobre el APL y el EPB son característicos del SI por el cruce de ambos sobre el extensor carpi radialis brevis y extensor carpi radialis longus. Aunque antes se consideraba que el mecanismo causante era el roce entre los extensores del pulgar con los tendones extensores de la muñeca, Grundberg y Reagan ${ }^{21}$ demostraron que la patología básica era la tenosinovitis del segundo compartimento extensor. Pero también es cierto que esa tenosinovitis adhiere el borde cubital del EPB.

d) Origen nervioso: Síndrome de Wartenberg (neuropatía compresiva de la rama sensitiva del nervio radial), en cuyo caso el dolor suele ser algo más proximal, en la confluencia del brachioradialis con el extensor carpi radialis longus, y cursa con hipoestesia y/o parestesias en el territorio radial, Finkelstein +, Brunelli - y Tinel + .

\section{TRATAMIENTO}

El tratamiento inicial de la TDQ debe ser conservador; lo que incluye la suspensión de la actividad causante cuando ésta se conoce (baja laboral), terapia con antiinflamatorios, inmovilización de la columna del pulgar con férulas, infiltración de corticoides y rehabilitación. Desde hace poco tiempo, con resultados aún no muy contrastados y a menudo poco alentadores, según nuestra experiencia personal, se emplea el tratamiento con ondas de choque.

Las infiltraciones de corticoides no están exentas de complicaciones, como hipopigmentación, necrosis cutánea y roturas tendinosas ${ }^{5,9,22}$, que en algún caso exigen un tratamiento quirúrgico de las mismas ${ }^{23}$. Deben realizarse las menos posibles (no parece aconsejable más de dos o tres $^{3,18}$ ), con una técnica rigurosa de aplicación y extremando las medidas de asepsia. Aun así muchas veces fracasan porque el corticoide pe- netra en la vaina del APL pero no en la del EPB cuando ambos están separados ${ }^{5}$.

Con estos métodos suelen curar alrededor del $30 \%$ al $60 \%$ de los $\operatorname{casos}^{18}$. Su fracaso indica el tratamiento quirúrgico, que suele consistir en abrir el retináculo. Pero esta única medida, aún teniendo la precaución de hacerlo por el borde más dorsal para evitar la luxación tendinosa, trae frecuentes complicaciones, de ahí que numerosos autores hayan propuesto la reconstrucción de la polea con diferentes técnicas.

No son infrecuentes las complicaciones del tratamiento quirúrgico, siendo las siguientes las más comunes:

a) Persistencia del dolor en el postoperatorio: Puede ser debida a una incompleta apertura cuando existen dos túneles osteofibrosos, a adherencias cicatriciales por material de sutura subcutánea o la inmovilización del pulgar tras la cirugía, a fibrosis perineural sobre las ramas sensitivas del nervio radial o a la concomitancia con un síndrome de intersección no diagnosticado previamente.

b) Luxación o subluxación del APL: Esta es una complicación frecuente tras la simple apertura de la polea. Para evitarla, gran parte de los autores aconsejan abrirla por su lado más dorsal dejando una franja de polea en el labio anterior de la corredera para que haga de tope. No obstante, la experiencia demuestra que esto es insuficiente en algunos casos; aún cuando los tendones no lleguen a luxarse, la carencia del retináculo produce un molesto efecto de arco de cuerda a menudo persistente, y en muchos pacientes el dolor postoperatorio con frecuencia no es ya sobre la apófisis estiloides como en el período preoperatorio, sino más distal, a unos $15 \mathrm{~mm}$, al hacer presión sobre el APL, en la oquedad radial del carpo, por subluxación de este tendón. La luxación franca del tendón se pone de manifiesto al pedir al paciente que abduzca el pulgar contra resistencia con la muñeca completamente flexionada.

c) Lesión del nervio radial: no son infrecuentes las lesiones iatrogénicas de las ramas sensitivas del nervio radial en el acto operatorio, complicación que debe evitarse identificando y diseccionando las ramas nerviosas antes de abordar la polea, y no ejerciendo sobre ellas una excesiva tracción. 
d) Lesión de la arteria radial: este vaso pasa bajo los tendones en la zona media de la tabaquera anatómica y puede lesionarse en las disecciones amplias y distales. e) Defectos estéticos: las incisiones longitudinales, perpendiculares a las líneas de Langer, pueden producir queloides o cicatrices hipertróficas.

\section{BIBLIOGRAFÍA}

1. De Quervain F. Ueber eine form von chronischer tendovaginitis. Korrespondenz-Blatt Schweizer Aerzrte 1895, 25: 389-94. (On a form of chronic tendovaginitis) Translated article in $\mathrm{J}$ Hand Surg B, 2005; 30: 388-91.

2. Ahuja NK, Chung KC, Arbor A. Fritz de Quervain MD (18681940): Stenosing tendovaginitis at the radial sytiloid process. $J$ Hand Surg Am, 2004; 29: 1164-70.

3. Phalen GS. Enfermedad de De Quervain. En Flynn JE. Cirugía de la Mano, $2^{\mathrm{a}}$ edición. Barcelona: Ed Toray SA, 1984: 505-8.

4. Savage RC. Enfermedad de De Quervain. En: McCarthy JG, May JW, Littler JW (Eds). Cirugía Plástica-La Mano, tomo I. Buenos Aires: Ed Médica Panamericana, 1992: 505-12.

5. Wolfe SW. Enfermedad de De Quervain. En Green's. Cirugía de la Mano, vol.2, Madrid: Ed Marbán, 2007: 2150-4.

6. Ceruso M. Artroplastiche biologiche. En La Rizoartrosi. Monografie della Società Italiana di Chirurgia della mano. Fidenza: Casa Editrice Mattioli, 1996: 133-49.

7. Zancolli E, Cozzi EP. Atlas de anatomía quirúrgica de la mano. Madrid: Ed. Médica Panamericana, 1993.

8. Bouchlis G, Bhatia A, Asfazadourian $\mathrm{H}$, et al. Distal insertions of abductor pollicis longus muscle and artritis of the first carpometacarpal joint in 104 dissections. Ann Chir Main, 1997; 16: 326-37.

9. Arroyo J, Delgado PJ, Fuentes A, et al. Tratamiento quirúrgico de la tenosinovitis estenosante de De Quervain. Patología del Aparato Locomotor, 2007: 5: 88-93.

10. Wetterkamp D, Rieger H, Brug E. Tratamiento quirúrgico de la tenosinovitis estenosante de De Quervain. Tech Quir Ortop Traumatol (edic esp.), 1999; 8: 109-14.

11. Arons MS. de Quervain's release in working women: A report of failures, complications, and associated diagnoses. J. Hand Surg Am, 1987; 12: 540-4.

12. Finkelstein $H$. Stenosing tenosynovitis at the radial styloid process. J Bone Joint Surg, 1930; 12: 509-40.

13. Brunelli G. Le test de Finkelstein contre le test de Brunelli dans la tenosynovite de De Quervain. Chir Main, 2003; 22: 43-5.

14. Eichhoff E. Zur pathogenese der tenovaginitis stenosans. Bruns Beitr Klin Chir, 1927; 139: 746-55.

15. Elliot BG. Finkelstein's test: a descriptive error that can produce a false positive. J Hand Surg B, 1992;17: 481-2.

16. Kutsumi K, Amadio PC, Zhao $\mathrm{Ch}$, et al. Finkelstein's test: a biomechanical analysis. J Hand Surg Am, 2005; 30: 130-5.

17. Le Viet D. Ténosynovite de De Quervain. Maîtrise Orthopédique 2002; 114:10-1.

18. Umlas ME y Gelberman RH. Tratamiento quirúrgico de la enfermedad de de Quervain. En: Gelberman RH. Muñeca. Madrid: Ed Marbán, 1999: 383-92.

19. Kaneko K, Matsumara K, Maruyama Y. Congenital synovitis between the scaphoid and the trapezium as a cause of tenosinovitis simulating de Quervain's disease. Ann Chir Main, 2000,19: 187-90.

20. Abe Y, Tsue K, Nagai E, et al. Extensor pollicis longus tenosinovitis mimicking de Quervain's disease because of its course through the first extensor compartment: a report of 2 cases. J Hand Surg Am, 2004; 29: 225-9.

21. Grundberg AB, Reagan DS. Pathologic anatomy of the forearm: Intersection syndrome. $J$ Hand Surg Am, 1985; 10: 229-302.

22. Yuen A, Coombs CJ. Abductor pollicis longus tendon ruptura in De Quervain's disease. J Hand Surg B, 2006; 31: 72-5.

23. González J, Del Valle B, López G. Una nueva técnica de tratamiento de la atrofia cutánea post-infiltración esteroidea en la muñeca. Rev Ortop Traum, 2001; 1: 56-60. 